\title{
LABORATORY CONTROL OF ANTICOAGULANT THERAPY THE EFFECT OF STORAGE ON BLOOD USED FOR PROTHROMBIN ESTIMATIONS
}

\author{
BY \\ W. LEWIN AND B. M. BLOOMBERG \\ With the Technical Assistance of IRENE MROST \\ From the Clinical Laboratories, Medical Centre, Johannesburg, South Africa
}

(RECEIVED FOR PUBLICATION JANUARY 24, 1957)

Suzman (1956) and Suzman, Ruskin, and Goldberg (1955) have confirmed that the incidence of repeated attacks of coronary thrombosis is less in patients undergoing prolonged anticoagulant therapy than in untreated cases. The main objection to anticoagulant therapy, however, is the necessity for strict laboratory control to avoid therapeutically induced haemorrhage. In an editorial in the Lancet (1956) it is stated that in "good risk" cases "the relatively small advantage to be obtained from anticoagulants must be set against the risk of transport to hospital; if the assessment is ' poor risk,' then the patient should be got to hospital for anticoagulant treatment as soon as possible." It appears from this statement that adequate control of anticoagulant therapy necessitates admission to hospital. We cannot agree that this is necessary or, in fact, advisable, our view being based on an experience extending over several years during which over 1,000 patients have been under laboratory control either ambulatory or domiciliary. Haemorrhage in adequately controlled cases has been rare. It has, however, hitherto apparently not been customary in England to undertake laboratory control in domiciliary practice. The major objections have been $(a)$ the fear of haemorrhage, which certainly occurred more frequently (Biggs and Macfarlane, 1949; James, 1949) when prothrombin estimations were performed with Russell's viper venom as the source of thromboplastin (Fullerton, 1940), and (b) the necessity of using freshly drawn blood for Quick, Stanley-Brown, and Bancroft's (1935) one-stage prothrombin test. The latter has apparently been based on Quick's (1951) statement that for research studies blood should be used within 30 minutes of collection, whereas up to four hours' storage was permissible for routine clinical purposes.

Since specimens submitted by distant medical practitioners could not reach our laboratories within four hours after collection, an investigation was undertaken in 1953 similar to that reported by Watson (1956), but no report was made at the time. However, in view of the increasing use of anticoagulants and of favourable recent reports (Suzman et al., 1955 ; Suzman, 1956 ; Wright, Marple, and Beck, 1948 ; Gilchrist and Tulloch, 1956), it was thought of interest to record a confirmation of Watson's findings. The main purpose of the study was to determine the effect of a 24hour delay on the estimation of the prothrombin index and to assess the significance of the possible loss of labile factor (Factor V).

\section{Methods}

Determination of Prothrombin Index.-This follows in its main essentials Stein's (1941) modification of the method of Quick et al. (1935). To duplicate samples of $0.1 \mathrm{ml}$. of plasma and $0.1 \mathrm{ml}$. of an approximately $6 \%$ solution of thromboplastin (prepared by incubating acetone-dried human brain with saline at $37^{\circ} \mathrm{C}$. for 15 minutes), $0.1 \mathrm{ml}$. of $\mathrm{M} / 40$ calcium chloride solution is added, a stop-watch started immediately, and the end-point determined as described by Stein (1941). We have preferred in routine practice to express our results as the "prothrombin index" (P.I.) which is obtained by dividing the prothrombin time of normal plasma by that of the patient's plasma and expressing the result as a percentage. The effect of adding labile factor or Factor $\mathrm{V}$ was studied by adding $0.1 \mathrm{ml}$. barium-sulphate-absorbed, i.e., prothrombin-free plasma prepared according to the directions of Rosenfield and Tuft (1947) to $0.9 \mathrm{ml}$. of test plasma. Samples each of $0.1 \mathrm{ml}$. of the mixture were then tested as described above.

\section{Discussion}

Like Watson (1956), little difference was found whether the sample of blood used for the estimation was fresh or 24 hours old. Stein (1941) had already suggested that plasma, if separated and kept in a cool place, may be used 24 hours later without showing much change in the prothrombin index, but this report has apparently 
been overlooked by later workers. Although Stein does not specifically state that the plasma remained corked after separation, he has confirmed (personal communication) that this was in fact done. This is of some significance as our experiments have demonstrated.

In the accompanying figures results are shown on 55 specimens, 49 of which were from patients on anticoagulant therapy. After estimating the prothrombin index on each specimen of blood, other samples of the same specimens were stored, corked, in a drawer which was opened and closed several times during the day to simulate conditions that might occur during the transporting of the specimens to the laboratory. After 24 hours under these conditions the mean difference in prothrombin times was only $0.7 \pm 1.4$ seconds. Fig. 1 shows that in only three specimens was an increase greater than 3 seconds obtained. When the actual prothrombin index is $50 \%$, which is the level aimed at in therapy, an increase of 3 seconds in the prothrombin time would mean that a prothrombin index of $45 \%$ is reported. At lower prothrombin indices, to produce an error of $5 \%$ would require even greater differences in prothrombin times, e.g., at a prothrombin index of $40 \%, 4$ seconds' difference is required, and at $30 \%$ 8 seconds' difference.

This error may be due either to inherent technical defects or to disappearance of labile clotting factors, e.g., Factor V, from the oxalated blood on standing. Either of these possibilities would

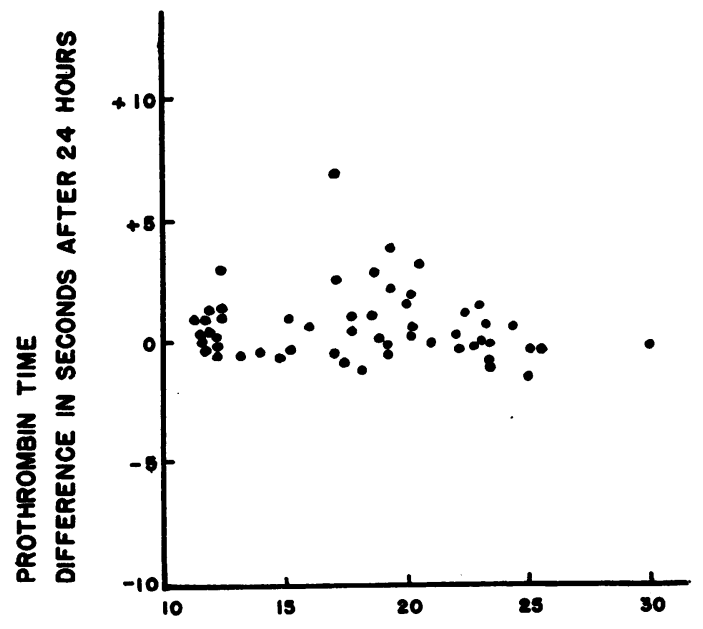

INITIAL PROTHROMEN TIME IN SECONDS

Fig. 1.-Differences in prothrombin times of oxalated plasma from venous blood stored in corked tubes at room temperature for 24 hours. (Mean difference \pm S.D. $=0.7 \pm 1.4$ seconds.)

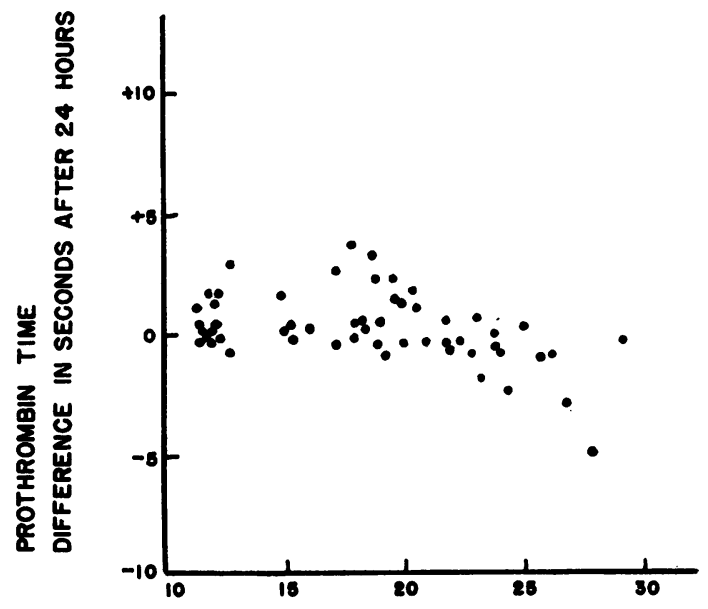

INITIAL PROTHROMBIN TIME IN SECONDS

Fig. 2.-Differences in prothrombin times after storage as in Fig. 1, and addition of labile factor.

fit the observed facts that the variations shown bear no constant relationship to the actual prothrombin times recorded from 12 seconds (prothrombin index $100 \%$ ) to 30 seconds (prothrombing index $40 \%$ ). Since anticoagulant therapy is known not to affect Factor V (Stefanini, 1950; Biggs anळ Macfarlane, 1953), it might be expected that destruction of the labile factor would be fairly uniform in all specimens and independent of the initial value of the prothrombin index. Since this could account for changes in the prothrombin times on standing over 24 hours, the effect of adding labile factor was determined. Fig. 2 shows that there is no decrease in the degree of scatter, suggesting that there has been no significant loss of the labile factor during storage of the blood under the conditions specified. The probability, therefore, remains that technical errors are responsible for the degree of scatter found.

When blood specimens are centrifuged soon after collection and the centrifuged blood allowed to stand in uncorked tubes at room temperature, considerable differences in prothrombin times (mean $6.5 \pm 7.4$ seconds) compared with whole blood are found after 24 hours. These are only partially corrected by adding labile factor (mean $3.0 \pm 5.5$ seconds). However, when the separated plasma is kept in corked tubes under similar conditions to whole blood there is much less change in the prothrombin times (mean $3.0 \pm 3.7$ seconds) in most of the specimens, but prothrombin time increases of over 5 seconds were observed in six specimens and over 10 seconds in two specimens. 
The addition of labile factor produced no significant change in the prothrombin times (mean $1.9 \pm 3.9$ seconds). Chak and Giri (1948) showed that the loss in prothrombin activity of plasma on storage could be restored by passing carbon dioxide through the plasma, and claimed that the carbon dioxide in some way neutralized the action of inhibitors which are formed during the period of storage. As, however, the prothrombin times after 24 hours of the separated plasma are slightly longer than those of plasma left in contact with their erythrocytes, both being kept corked under similar conditions, Stein and Abrahams (personal communication) have suggested that the red cells exert a buffering action and thus retard the escape of carbon dioxide.

\section{Summary}

A report is given of the laboratory control of domiciliary and ambulatory cases on prolonged anticoagulant therapy. Several of these patients have been under treatment for over five years. In the authors' experience, which extends to over
1,000 patients on prolonged anticoagulant therapy, haemorrhage in adequately controlled cases is rare. The prothrombin index, performed on samples of oxalated venous blood, does not appreciably vary for 24 hours after withdrawal, provided the specimens are maintained corked. No relationship could be established between the variations found and possible deterioration of labile factor, and such variations are put down to experimental error.

\section{REFERENCES}

Biggs, R., and Macfarlane, R. G. (1949). J. clin. Path., $2,33$. Biggs, R., and Macfarlane, R. G. (1953). Human Blood Coagulation and its Disorders. Blackwell, Oxford

Chak, I. M., and Giri, K. V. (1948). Nature (Lond.), 161, 354.

Editorial (1956). Lancet, 1, 559 .

Fullerton, H. W. (1940). Ibid., 2, 195.

Gilchrist, A. R., and Tulloch, J. A. (1956). Scottish med. J., 1, 1 James, G. A. (1949). J. clin. Path., 2, 45.

Rosenfield, R. E., and Tuft, H.S. (1947). Amer.J.clin. Path., 17, 405. Quick, A. J. (1951). The Physiology and Pathology of Hemostasis, p. 127. Kimpton, London.

Stanley-Brown, M., and Bancroft, F. W. (1935). Amer. J. med. Sci., 190, 501.

Stefanini, M.(1950). Amer. J. clin. Path., $20,233$.

Stein, H. B. (1941). S. Afr. J. med. Sci.,6, 93 .

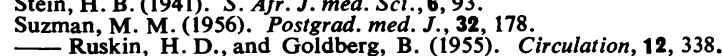
Watson, H. (1956). Lancet, 1, 541.

Wright, I. S., Marple, C. D., and Beck, D. F. (1948). Amer. Heart J., 36, 801 . 